\title{
Prioritization of rehabilitation interventions for urban water assets using multiple criteria decision-aid methods
}

\author{
N. Carriço, D. I. C. Covas, M. Céu Almeida, J. P. Leitão and H. Alegre
}

\begin{abstract}
The aim of this paper is to compare sorting and ranking methods for prioritization of rehabilitation interventions of sewers, taking into account risk, performance and cost. For that purpose multiple criteria decision-aid (MCDA) methods such as ELECTRE TRI for sorting and ELECTRE III for ranking are applied in a real case-study and the results obtained are compared. The case study is a small sanitary sewer system from a Portuguese utility located in the metropolitan area of Lisbon. The problem to investigate is the prioritization of the sewer candidates for rehabilitation. The decision maker (a panel group of specialists) has chosen five assessment measures: water level and maximum flow velocity (hydraulic performance indices), sewer importance and failure repair cost (collapse-related consequences of failure) and the risk of collapse. The results show that the

N. Carriço (corresponding author) D.I. C. Covas

Instituto Superior Técnico (IST) Avenida Rovisco Pais, 1, 1049-001 Lisboa,

Portugal

E-mail: nelson.carrico@gmail.com

M. Céu Almeida

J. P. Leitão

H. Alegre

Laboratório Nacional de Engenharia Civil (LNEC), Avenida do Brasil, 101,

1700-066 Lisboa,

Portugal outcomes from ELECTRE III are easier to understand than those from ELECTRE TRI method. Two different sets of weights were used, and the sorting and ranking results from both methods were found to be sensitive to them. ELECTRE TRI method is not straightforward as it involves technical parameters that are difficult to define, such as reference profiles and cut levels.

Key words $\mid$ cost, MCDA, performance, prioritization, rehabilitation plan, risk
\end{abstract}

